\title{
County-Level Association of Social Vulnerability with COVID-19 Cases and Deaths in the USA
}

J Gen Intern Med 35(9):2784-7

DOI: $10.1007 / \mathrm{s} 11606-020-05882-3$

(c) Society of General Internal Medicine 2020

\section{INTRODUCTION}

In past pandemics, vulnerable populations faced greater disease burden and decreased testing and treatment access. ${ }^{1}$ As coronavirus disease 2019 (COVID-19) spreads in the USA, concern is growing that even the early stages of this pandemic have disproportionately impacted vulnerable communities. ${ }^{2-4}$ However, the relationship between social vulnerability and COVID-19 diagnosis and mortality in rural and urban communities remains unknown.

\section{METHODS}

We performed a county-level, cross-sectional analysis using COVID-19 case and death rates compiled by The New York Times from health agency reports as of April 19, 2020. We stratified counties into quartiles using the U.S. Centers for Disease Control's Social Vulnerability Index (SVI), a validated measure of community resilience during natural disasters and disease outbreaks across four domains: socioeconomic status, household composition and disability, minority status and language, and housing and transportation. ${ }^{5} \mathrm{We}$ defined urbanicity using the U.S. Department of Agriculture Economic Research Service's 2013 Urban Influence Codes. ${ }^{6}$ We merged data sources using Federal Information Processing Standard (FIPS) codes, including counties with a linkable FIPS code and at least one COVID-19 case.

Our primary outcomes were positive tests per capita and COVID-19 deaths per capita. We built populationweighted, quasi-Poisson regression models to compare outcomes between the first and fourth quartiles of counties by SVI and each SVI domain. In secondary analyses, we stratified counties by rural and urban classification. We included state fixed effects to account for

Rohan Khazanchi and Evan R. Beiter contributed equally to this work.

Received April 23, 2020

Accepted April 28, 2020

Published online June 23, 2020 heterogeneity in policies and disease spread. We analyzed data with $\mathrm{R}$ Statistical Software, version 3.6.3, and considered $P<0.002$ significant after the Bonferroni correction. This study was approved by Partners Healthcare Institutional Review Board.

\section{RESULTS}

As of April 19, there were 612,404 confirmed cases and 25,978 COVID-19 deaths across the 2754 (of 3143 total) counties analyzed (mean cases 102.2 per 100,000 [SE 3.8], deaths 4.0 per 100,000 [0.2]). Compared with those in the least vulnerable counties, people in the most vulnerable counties had 1.63 -fold greater risk of COVID-19 diagnosis and 1.73-fold greater risk of death (Table 1). When considering only the minority status and language domain, people in the most vulnerable counties had 4.94-fold and 4.74-fold greater risks of COVID-19 diagnosis and death, respectively. Mapping case burden in the most and least vulnerable counties by minority status revealed regional trends of this differential risk (Fig. 1). Similarly, people in the most vulnerable counties by socioeconomic status (relative risks [RR] of 1.42 and 1.71) and housing and transportation (RR 1.52 and 1.32) domains had greater risk of COVID-19 diagnosis and death. Vulnerability by the household composition and disability domain was not associated with differential risk.

These trends persisted among urban counties alone. Among rural counties alone, the most vulnerable counties by minority status and language had greater risk of COVID-19 diagnosis (RR 3.74), while associations with overall SVI, socioeconomic status, and housing and transportation were no longer significant.

\section{DISCUSSION}

Greater social vulnerability is associated with increased risk of COVID-19 detection and death. In urban and rural counties alike, this is driven by differences across the minority status and language domain, consistent with preliminary reports of increased COVID-19 prevalence and mortality among minorities. ${ }^{2}$ Factors such as poverty, unemployment (socioeconomic status domain), crowded housing, and vehicle access (housing and 
Table 1 COVID-19 Cases and Deaths per Capita by Most and Least Socially Vulnerable Urban and Rural Counties

\begin{tabular}{|c|c|c|c|c|c|}
\hline & & Q1 & Q4 & Relative risk $^{\dagger}$ & $95 \% \mathrm{CI}$ \\
\hline Social Vulnerability Index & $n=$ & 634 & 723 & & \\
\hline \multirow[t]{2}{*}{ Total } & Cases per 100,000 & $92.9(6.6)$ & $122.9(7.6)$ & $1.63^{*}$ & $1.49-1.78$ \\
\hline & Deaths per 100,000 & $3.71(0.39)$ & $5.42(0.55)$ & $1.73 *$ & $1.55-1.93$ \\
\hline Urban $^{\S}$ & Cases per 100,000 & $119.7(11.7)$ & $166.2(18.0)$ & $1.77 *$ & $1.57-2.00$ \\
\hline $\mathrm{Q} 1=315, \mathrm{Q} 4=202$ & Deaths per 100,000 & $4.94(0.64)$ & $7.39(1.23)$ & $1.87 *$ & $1.60-2.17$ \\
\hline $\operatorname{Rural}^{\S}$ & Cases per 100,000 & $66.3(5.9)$ & $106.2(7.9)$ & 0.92 & $0.68-1.24$ \\
\hline $\mathrm{Q} 1=319, \mathrm{Q} 4=521$ & Deaths per 100,000 & $2.49(0.43)$ & $4.66(0.59)$ & 0.66 & $0.36-1.19$ \\
\hline Socioeconomic status $\|$ & $n=$ & 647 & 710 & & \\
\hline \multirow[t]{2}{*}{ Total } & Cases per 100,000 & $102.8(7.4)$ & $112.4(7.1)$ & $1.42 *$ & $1.26-1.60$ \\
\hline & Deaths per 100,000 & $3.74(0.37)$ & $5.08(0.54)$ & $1.71 *$ & $1.47-1.98$ \\
\hline Urban & Cases per 100,000 & $130.1(11.3)$ & $148.8(17.8)$ & $1.61 *$ & $1.36-1.91$ \\
\hline $\mathrm{Q} 1=351, \mathrm{Q} 4=163$ & Deaths per 100,000 & $5.12(0.59)$ & $6.67(1.35)$ & $1.86^{*}$ & $1.50-2.30$ \\
\hline Rural & Cases per 100,000 & $70.4(8.8)$ & $101.5(7.4)$ & 0.64 & $0.44-0.94$ \\
\hline $\mathrm{Q} 1=296, \mathrm{Q} 4=547$ & Deaths per 100,000 & $2.11(0.39)$ & $4.61(0.57)$ & 0.77 & $0.36-1.62$ \\
\hline \multirow{3}{*}{$\begin{array}{l}\text { Household composition and disability } \\
\text { Total }\end{array}$} & $n=$ & 687 & 691 & & \\
\hline & Cases per 100,000 & $131.3(9.8)$ & $100.8(6.7)$ & 0.85 & $0.73-0.99$ \\
\hline & Deaths per 100,000 & $4.81(0.44)$ & $4.62(0.53)$ & 1.10 & $0.93-1.30$ \\
\hline Urban & Cases per 100,000 & $158.9(14.0)$ & $125.8(16.4)$ & 1.00 & $0.79-1.28$ \\
\hline $\mathrm{Q} 1=436, \mathrm{Q} 4=169$ & Deaths per 100,000 & $6.24(0.63)$ & $6.24(1.26)$ & 1.29 & $1.01-1.66$ \\
\hline Rural & Cases per 100,000 & $83.4(10.2)$ & $92.7(7.1)$ & 1.05 & $0.82-1.35$ \\
\hline $\mathrm{Q} 1=251, \mathrm{Q} 4=522$ & Deaths per 100,000 & $2.32(0.47)$ & $4.09(0.57)$ & 1.42 & $0.95-2.12$ \\
\hline \multirow{3}{*}{$\begin{array}{l}\text { Minority status and language } \\
\text { Total }\end{array}$} & $n=$ & 625 & 706 & & \\
\hline & Cases per 100,000 & $51.2(2.6)$ & $158.1(11.1)$ & $4.94 *$ & $3.91-6.24$ \\
\hline & Deaths per 100,000 & $2.11(0.24)$ & $5.86(0.52)$ & $4.74 *$ & $3.55-6.32$ \\
\hline Urban & Cases per 100,000 & $54.1(3.8)$ & $203.2(18.0)$ & $5.02 *$ & $3.20-7.88$ \\
\hline $\mathrm{Q} 1=162, \mathrm{Q} 4=377$ & Deaths per 100,000 & $1.88(0.24)$ & $8.14(0.85)$ & $5.30 *$ & $3.03-9.28$ \\
\hline Rural & Cases per 100,000 & $50.2(3.2)$ & $106.4(11.4)$ & $3.74 *$ & $2.66-5.25$ \\
\hline $\mathrm{Q} 1=463, \mathrm{Q} 4=329$ & Deaths per 100,000 & $2.18(0.31)$ & $3.25(0.48)$ & 1.60 & $0.88-2.93$ \\
\hline \multirow{3}{*}{$\begin{array}{l}\text { Housing type and transportation } \\
\text { Total }\end{array}$} & $n=$ & 612 & 730 & & \\
\hline & Cases per 100,000 & $82.1(4.7)$ & $140.1(10.0)$ & $1.52 *$ & $1.35-1.72$ \\
\hline & Deaths per 100,000 & $2.84(0.28)$ & $5.81(0.58)$ & $1.32 *$ & $1.14-1.53$ \\
\hline Urban & Cases per 100,000 & $100.8(7.4)$ & $190.4(21.0)$ & $1.53 *$ & $1.30-1.81$ \\
\hline $\mathrm{Q} 1=291, \mathrm{Q} 4=286$ & Deaths per 100,000 & $3.84(0.44)$ & $7.42(1.03)$ & $1.29 *$ & $1.05-1.59$ \\
\hline Rural & Cases per 100,000 & $65.1(5.7)$ & $107.7(8.9)$ & 1.08 & $0.84-1.37$ \\
\hline $\mathrm{Q} 1=321, \mathrm{Q} 4=444$ & Deaths per 100,000 & $1.92(0.34)$ & $4.77(0.67)$ & 1.27 & $0.87-1.84$ \\
\hline
\end{tabular}

$Q 1=$ least vulnerable quartile; $Q 4=$ most vulnerable quartile. $Q 1$ and $Q 4$ are reported as mean (SE) values

*Statistically significant result, based on $P<0.002$ after Bonferroni correction

${ }^{\dagger}$ Relative risk was calculated from population-weighted, quasi-Poisson regression models with state fixed effects

${ }^{7}$ The Social Vulnerability Index is an aggregate of all four domains, each calculated based on variables from the 2014-2018 US Census American Community Survey data

${ }^{\xi}$ Urban and rural characteristics were determined from the U.S. Office of Management \& Budget categorization of the U.S. Department of Agriculture Economic Research Service's 2013 Urban Influence Codes, with a code of 1-2 (i.e., metropolitan areas) classified as "Urban" and a code of 3-12 (i.e., non-metropolitan areas) classified as "Rural"

"The socioeconomic status domain includes income, poverty, employment, and education variables

"The household composition and disability domain includes dependent children less than 18 years of age, persons 65 and older, single-parent households, and people with disabilities

${ }^{7}$ The minority status and language domain includes race, ethnicity, and English language proficiency variables

${ }^{\#}$ The housing type and transportation domain includes housing structure, crowding, and vehicle access variables

transportation domain) were associated with increased COVID-19 diagnosis and mortality in urban areas.

In rural communities, the minority status and language domain persists as a driver of increased COVID-19 cases. The disproportionate impact of COVID-19 on minority and non-English-speaking communities in both urban and rural areas may reflect compounding effects of structural racism, increased burden of chronic disease risk factors, and health care access barriers.

This cross-sectional, county-level study does not allow for causal, individual-level inferences. Analyses did not account for all county-level differences in testing rates or pandemic progression, although state fixed effects accounted for some geographic heterogeneity. As case reporting improves, analyzing more granular groupings of non-metropolitan counties may further elucidate rural trends.

In light of planned federal guidelines for county-level COVID-19 risk stratification and limited national demographic data, ${ }^{4}$ our findings reemphasize the need for standardized collection of sociodemographic characteristics. Targeted interventions addressing geographically variable social vulnerabilities may be necessary to improve inequitable outcomes of the COVID-19 pandemic, and health disparities more broadly. 
Geographic variation in COVID-19 cases per capita between most and least socially vulnerable counties by minority status and language
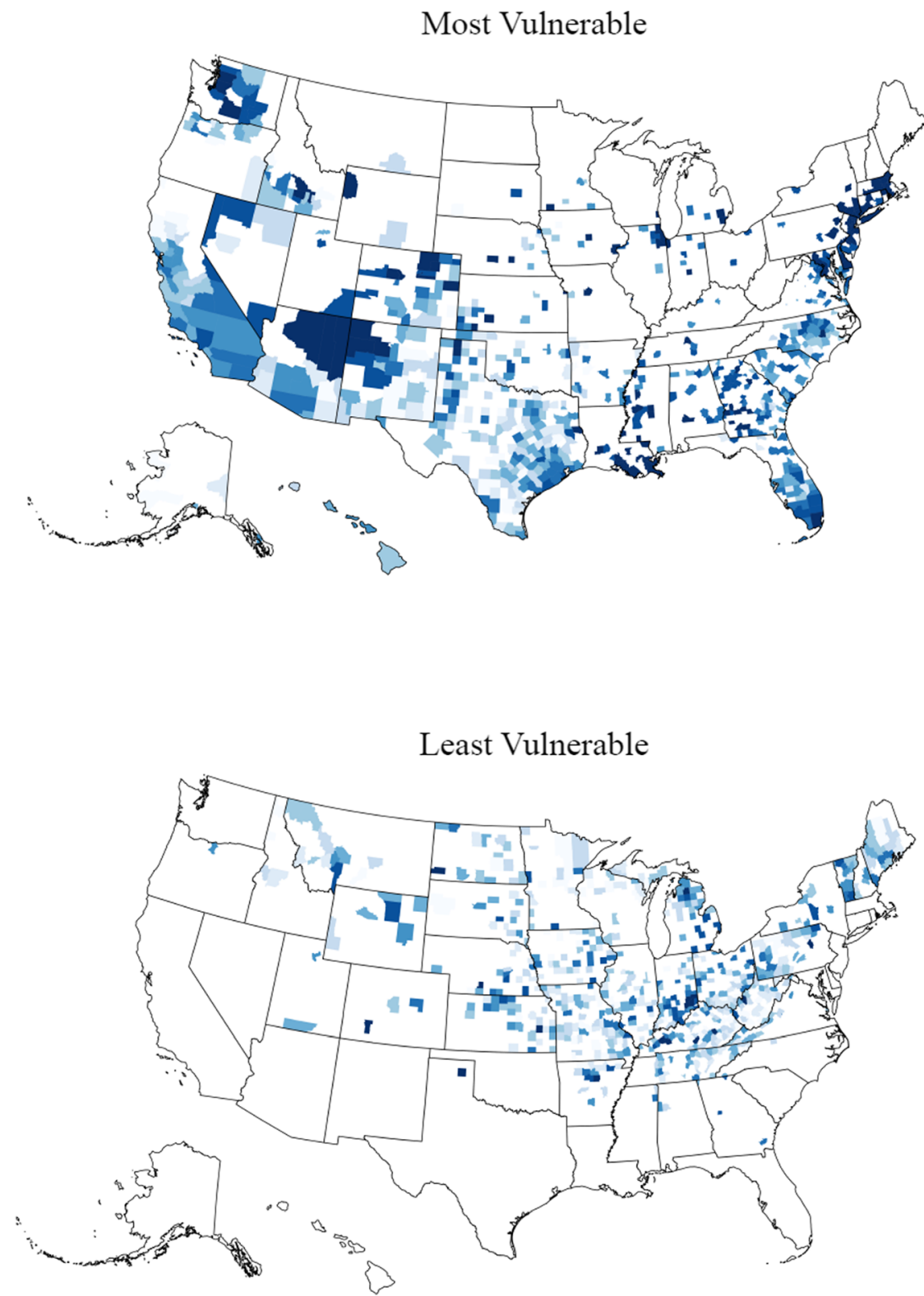

COVID-19 cases

per 100,000 people

$[1.56,10.8)$

$[10.82,19.4)$

$[19.41,29.4)$

$[29.45,38.5)$

$[38.47,54.0)$

$[54.00,73.9)$

$[73.94,108.9)$

$[108.95,215.8)$

$[215.79,2875.1]$

Figure 1 The most vulnerable quartile of counties $(n=706$, top $)$ and the least vulnerable quartile of counties $(n=625$, bottom), as indicated by the minority status and language domain of the U.S. Centers for Disease Control's Social Vulnerability Index. ${ }^{5}$ Counties without linked FIPS code or reported COVID-19 cases were excluded. Darker shades represent counties with more cases per capita.

Rohan Khazanchi, $B A^{1}$

Evan R. Beiter, $B A^{2}$

Suhas Gondi, $B A^{2}$

Adam L. Beckman, BS ${ }^{2}$

Alyssa Bilinski, $\mathrm{MS}^{3}$

Ishani Ganguli, $M D, M^{2} H^{2,4}$

${ }^{1}$ University of Nebraska Medical Center,

Omaha, NE, USA
${ }^{2}$ Harvard Medical School,

Boston, MA, USA

${ }^{3}$ Harvard Graduate School of Arts and Sciences,

Boston, MA, USA

${ }^{4}$ Division of General Internal Medicine and

Primary Care, Department of Medicine,

Brigham \& Women's Hospital,

Boston, MA, USA 
Corresponding Author: Ishani Ganguli, MD, MPH; Division of General Internal Medicine and Primary Care, Department of Medicine, Brigham \& Women's Hospital Boston, MA, USA (e-mail: iganguli@bwh.harvard.edu).

Data Availability The datasets analyzed during the current study are readily available from the following public repositories. NYTimes: https://github.com/nytimes/covid-19-data, USDA ERS Urban Influence Codes: https://www.ers.usda.gov/dataproducts/urban-influence-codes/, CDC SVI: https://svi.cdc.gov/ data-and-tools-download.html, USDA County FIPS Codes: https://www.nrcs.usda.gov/wps/portal/nrcs/detail/national/ home/?cid=nrcs143_013697

\section{Compliance with Ethical Standards:}

This study was approved by Partners Healthcare Institutional Review Board.

Conflict of Interest: Dr. Ganguli reports consulting fees from Haven and Blue Cross Blue Shield Massachusetts for work unrelated to this research. All authors submitted ICMJE Conflict of Interest forms, and no other authors report relevant conflicts of interest.

\section{REFERENCES}

1. Guinn SC, Kumar S, Freimuth VS, Musa D, Casteneda-Angarita N, Kidwell K. Racial Disparities in Exposure, Susceptibility, and Access to Health Care in the US H1N1 Influenza Pandemic. Am J Public Health. 2011;101(2):285-293. doi:https://doi.org/10.2105/AJPH.2009.188029

2. Yancy CW. COVID-19 and African Americans. JAMA. 2020. doi:https:// doi.org/10.1001/jama.2020.6548

3. Scigliano E. 'It Really Is the Perfect Storm': Coronavirus Comes for Rural America. POLITICO. https://www.politico.com/news/magazine/2020/04/ 15/coronavirus-rural-america-covid-19-186031. Published April 15, 2020. Accessed Apr 20, 2020.

4. Krieger N, Gonsalves G, Bassett MT, Hanage W, Krumholz HM. The Fierce Urgency Of Now: Closing Glaring Gaps In US Surveillance Data On COVID-19. Health Aff Blog. 2020. doi:https://doi.org/10.1377/ hblog20200414.238084

5. Centers for Disease Control and Prevention. CDC's Social Vulnerability Index. https://svi.cdc.gov/. Published September 5, 2018. Accessed Apr 2, 2020.

6. USDA ERS - Urban Influence Codes. https://www.ers.usda.gov/dataproducts/urban-influence-codes/. Accessed Apr 20, 2020.

Publisher's Note: Springer Nature remains neutral with regard to jurisdictional claims in published maps and institutional affiliations. 